\title{
Results Of Colon Anastomosis Formation After Sigmoid Colon Resection
}

Ten Dmitry Olegovich,

Doctor Of Philosophy In Medical Sciences (Phd), Head Of The Department Of Surgical Diseases, Fergana Branch Of The Tashkent Medical Academy, Uzbekistan

\section{G OPEN ACCESS}

The American Journal of Medical Sciences And Pharmaceutical

Research

JULY 2020

Page No.: 31-37

Volume-II Issue-VII

PUBLISHED: 30 JULY 2020

www.usajournalshub.com/inde

x.php/TAJMSPR

Copyright: Original content

from this work may be used

under the terms of the

Creative Commons Attribution

4.0 licence.

\section{Abstract}

An experimental study of the improved methods of colonic anastomoses on 30 mongrel dogs was carried out. The study showed that the use of the methods of colonic anastomoses developed by us does not lead to postoperative complications (anastomotic leakage, narrowing of the anastomotic area, etc.), is easily feasible, does not require expensive equipment, and can be performed in any surgical department.

Keywords: anastomosis, intestinal obstruction, volvulus of the sigmoid colon, bowel resection, sigmoid colon.

\section{Introduction}

Today, the issue of preventing such a formidable complication as insufficient anastomosis sutures remains one of the unsolved problems, both in urgent and in elective bowel surgery. According to the literature, the incidence of intestinal suture failure varies from $0.3-18.7 \%$ of observations, and under peritonitis conditions reaches $34.2 \%$ and has no tendency to decrease. Despite numerous attempts to improve the technical options for anastomosis, today it is not possible to overcome the negative aspects of these methods of joining intestinal integrity, dissatisfaction with the results forces us to return again to the problem of the intestinal suture, first of all, from the standpoint of quality and reliability $[2,4,5,9,10]$.

The use of modern stapling devices reduced the time of the operation, but did not 
significantly reduce the number of postoperative complications, so the inconsistency of the sutures ranges from $3.5 \%$ to $6.4 \%$. In the daily practice of a surgeon, it is not possible to widely introduce new technologies that improve the quality of the intestinal suture due to the high cost of apparatus and devices for the formation of anastomoses. In the conditions of urgent surgery, until now the dominant hand suture is, which is constantly being improved, and in combination with modern suture materials it allows to reduce the incidence of anastomotic suture failure $[1,2,9,11]$.

Despite the improvement of the technique of applying manual and mechanical colonic anastomoses, the high quality of modern suture materials and staplers, today it is not possible to overcome the negative aspects of these methods of anastomosis: the presence of foreign inclusions in the tissue junction area lengthens the regeneration time, the formation of wound channels promotes the penetration of endogenous microflora into the deep structures of the anastomosis and causes the development of an inflammatory reaction followed by cicatricle deformation of the anastomosis $[3,4,7,8,10,11]$.

In connection with the above, we set a goal: to improve and substantiate the imposition of methods of colonic anastomoses.

\section{Material And Research Methods}

We have carried out experimental studies on 30 mongrel dogs of both sexes, weighing 15$20 \mathrm{~kg}$. The main method of anesthesia is intravenous anesthesia using sodium thiopental 5-6 mg / kg. After thorough treatment of the operating field with alcohol and iodine, a median laparotomy was performed. The first stage of the operation was to create an acute obstruction of the sigmoid colon (ligation of the sigmoid colon). At the second stage, after 12-14 hours, the altered part of the sigmoid colon was resected, followed by the imposition of two types of colonic anastomosis according to our proposed technique. The technique of colonic anastomosis is as follows: after resection of the affected area of the intestine, a cuff was created from the mucous membrane of the adducting part of the intestine. After that, the walls of the anastomosis were formed with two different singlerow sutures ((№IAP 04333 and №IAP 04334 State Patent Office of the Republic of Uzbekistan). The operation ended with sanitation of the abdominal cavity. The postoperative wound was sutured tightly.

\section{Results And Discussion}

An analysis of the results of the experimental study showed that on the 2 nd day after the operation, motor activity was manifested in the operated dogs. On the 2-3rd day, the bowel function returned to normal, and its emptying was noted. On the 5th day, the dogs were transferred to a normal diet. In all operated dogs, the healing of the postoperative wound occurred by primary intention. The postoperative period was uneventful (all dogs received antibiotic therapy), we did not observe mortality after the operation.

The observation of the operated dogs continued for three months after the operation. Withdrawal from the experiment was carried out on 7, 14, 20 days, 1, 2, 3 months.

We did not observe pathological changes in the abdominal organs during autopsy of the operated dogs. By revising the intestine, we found a site of the anastomosis, which macroscopically differed little from other areas of the large intestine. On days 7, 14, 20, 
upon opening the macro-preparation, the cuff we created remained soft, viable, pale pink in color, there was no narrowing of the intestinal lumen. After 30 days, a mature connective tissue scar was formed at the site of the anastomosis suture; it was not possible to determine the boundaries of the cuff, due to complete fusion with the intestinal mucosa, the proposed border of the cuff was pale pink. The site of the anastomosis was excised over a length (adducting department-10 cm, ejection-10 cm) from several sites of the anastomosis, a biopsy material was taken, which was subjected to histological examination.

When using vicryl and a continuous suture, detritus and fibrin are not detected on the surface. There are pronounced processes of neovasculogenesis (Pic. 1, 2).

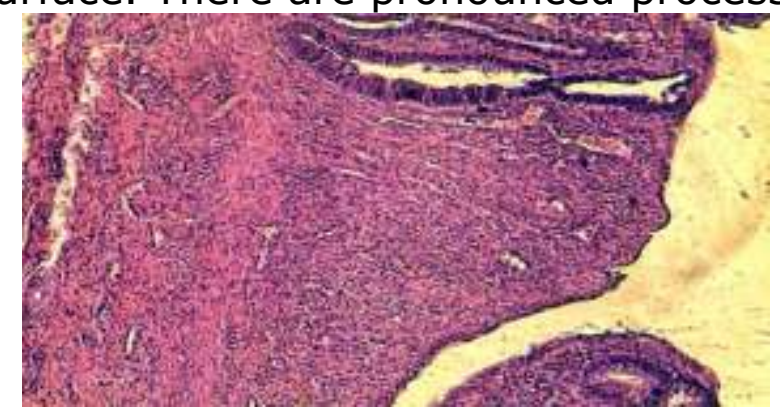

$10 \times 10$

Pic. 1. Joint area when applying a continuous suture using vicryl. H-E

In the early stages after the suture was applied, both nodular with the help of nylon and silk, and continuous with the use of vicryl, the growth of granulation connective tissue with a large number of microvessels was noted in the joint area. The crypts adjacent to the junction are expanded. The stroma is edematous and infiltrated with a large number of cells, mainly lymphocytes.

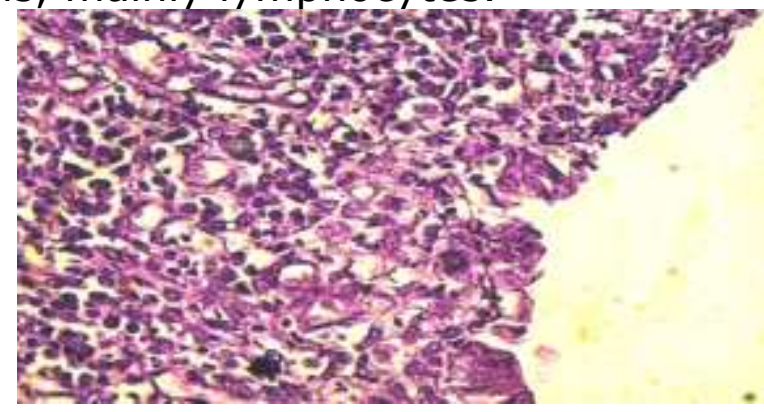

Pic. 2. Granulation tissue with microvessels and round cell elements. Joint area when applying a continuous seam using Vicryl H-E 10x40

When using an interrupted suture with nylon threads, small accumulations of fibrin with migrated lymphocytes are observed on the surface of the granulation tissue (Pic. 3 ).

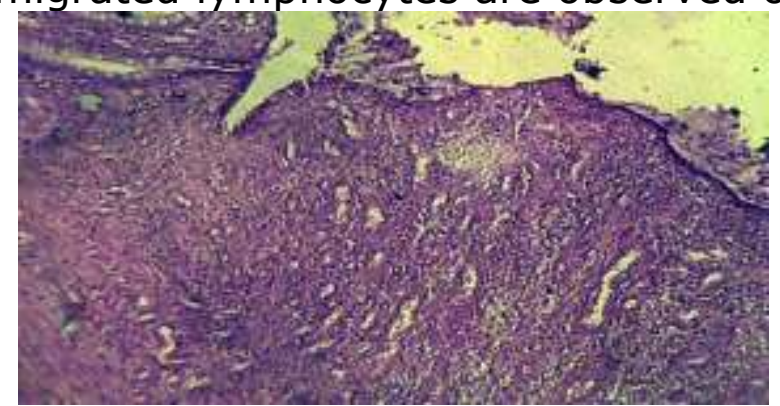

Pic. 3. The area of the joint when applying interrupted sutures using nylon 


\section{H-E $10 \times 10$}

These processes are especially pronounced when applying a knot suture and using silk (Pic.4).

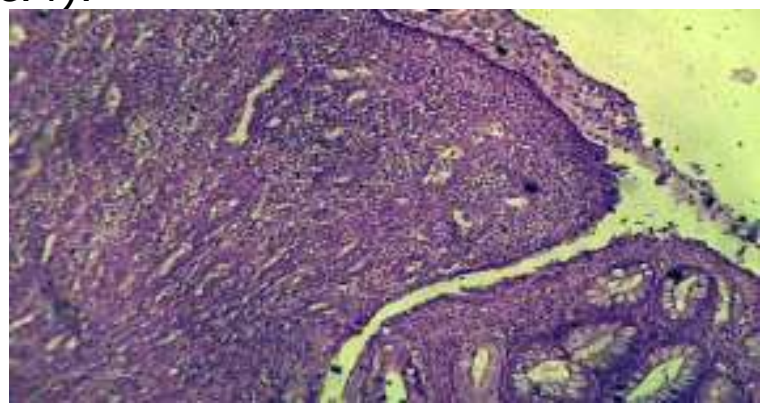

\section{Pic. 4. The joint area when applying interrupted sutures with silk.}

\section{H-E $10 \times 10$}

In later periods of observation, pronounced processes of creeping of the tongues of epithelial cells onto the surface of granulation tissue at the junction of the proximal and distal segments of the intestine are noted.

These processes are more pronounced when using interrupted sutures with vicryl. At the same time, not only the creeping of epithelial tongues onto the granulation surface free of fibrin and detritus is noted, but the immersion of epithelial strands into the granulation tissue, with the formation of crypts (Pic. 5, 6).

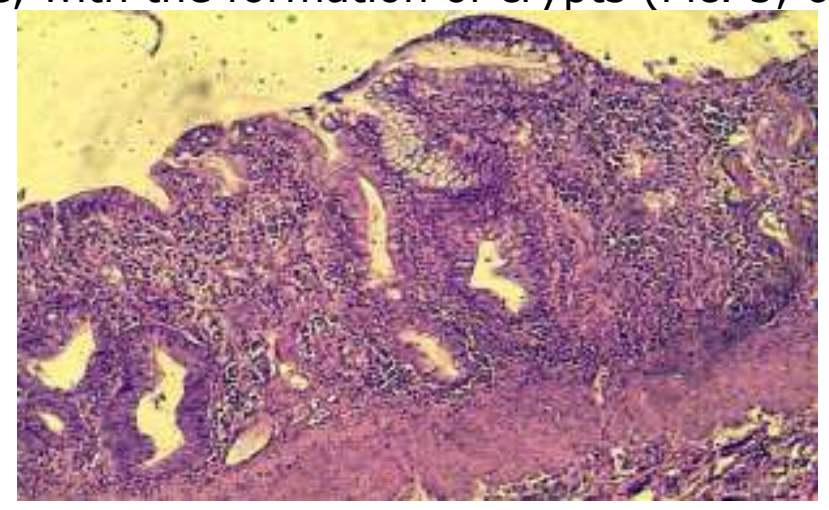

Pic. 5. Epithelization of the granulation tissue of the junction area when applying a continuous suture using Vicryl H-E $10 \times 10$

When anastomosis is applied with the help of interrupted sutures, the epithelial tongues are formed in the enlarged areas of the crypts adjacent to the granulation tissue at the junction of adjacent sections of the intestine (Pic. 6).

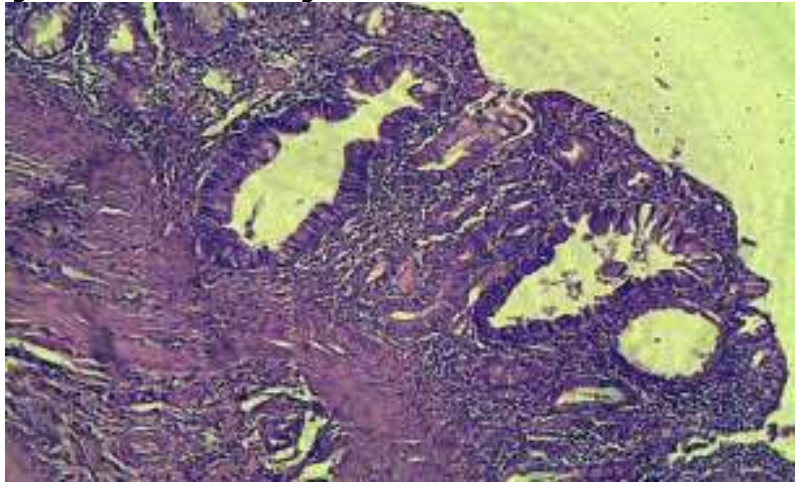

Pic. 6. Epithelialization of granulation tissue with the formation of crypts of 
ISSN (e): 2689-1026

DOI: https://doi.org/10.37547/TAJMSPR/Volume02Issue07-05

the junction area when applying a continuous suture using Vicryl H-E 10x10

The creeping of epithelial tongues onto the surface of granulations in the area of joints is not accompanied by a pronounced submerged growth of epithelial tongues with the formation of crypts, especially when silk is used (Pic. 6-8).

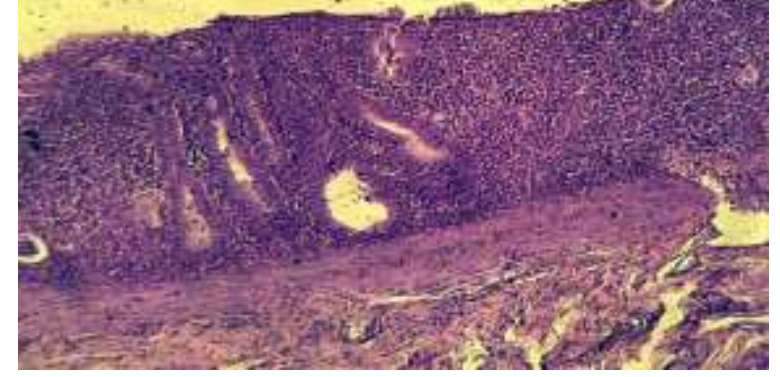

Pic. 7. Weakly expressed processes of epithelialization with manifestations of an inflammatory reaction of the joint area when applying an interrupted suture using silk H-E $10 \times 10$

At the same time, the muscle layer of the mucous membrane is being restored. At the same time, the vector orientation of the layers of smooth muscles is not determined in it (Pic. 6-8). At this stage, they are located chaotically, especially when using silk with interrupted sutures (Pic. 9).

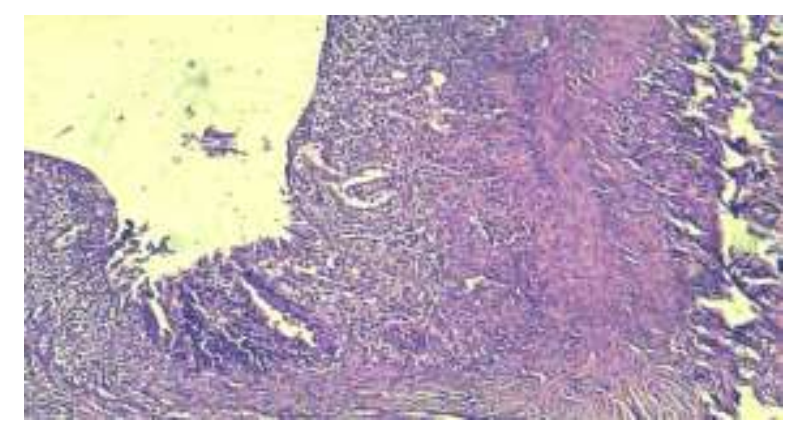

Pic. 8. Weakly expressed epithelialization with manifestations of an inflammatory reaction of the joint area when applying an interrupted suture using silk H-E 10x10

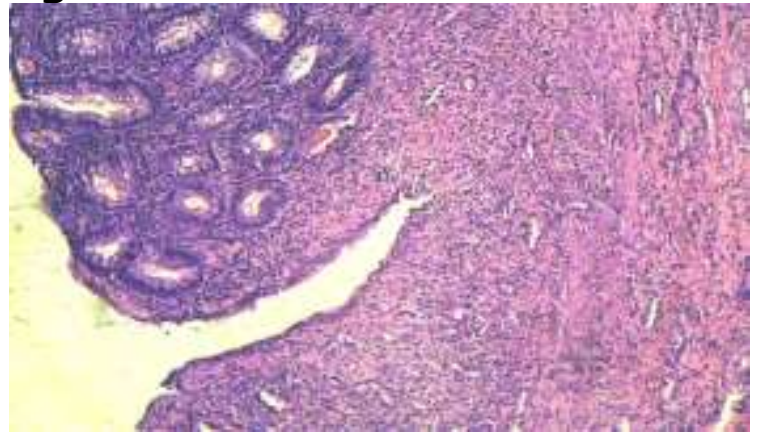

Pic. 9. Chaotic arrangement of smooth muscles in the joint area when applying an interrupted suture using nylon H-E $10 \times 10$

A rather large number of microvessels remains in the granulation tissue, but the number of cellular elements decreases, which is especially pronounced when using vicryl and a continuous suture (Pic. 5,6).

As the epithelial strands crawl onto the granulation surface, the lumens of the 
nearby crypts decrease somewhat, but their tortuosity and significant infiltration of the intercriptal connective tissue by polymorphic elements remain (Pic. 9).

\section{Conclusions}

To Sum up the results of our research, we made the following conclusions:

1. Conducted morphological studies have shown certain advantages of a continuous suture using vicryl, in comparison with interrupted sutures and the use of nylon and, especially, silk. With a continuous suture, the joint surface is cleared of detritus and fibrin faster epithelialization processes with the formation of crypts are faster on the formed granulation tissue.

2. The use of the methods of colonic anastomoses that, we have developed do not lead to postoperative complications (anastomotic failure, narrowing of the anastomotic area, etc.).

3. The use of the methods of colonic anastomoses we have proposed is easy to perform, does not require expensive equipment, and can be performed in any surgical department.

\section{References}

1. Buyanov V.M; Egiev V.N; Udotov O.A. Surgical suture. // Monograph. MedpraktikaM. 2001. -p. 109.

2. Esin V.L; Silischev R.V; Grishin K.N. Choice of colonic anastomosis. // Actual problems of coloproctology Abstracts of the IV Conf. Irkutsk: 1999. pp. 36-37.

3. Karimov Sh.I; Asrarov A.A; Orzimatov S.K; Babakhanov O.A. The role of enteral tube feeding in the treatment of patients with acute intestinal obstruction. // Surgery of Uzbekistan 2004. No. 2. -pp. 32-37.

4. Krasilnikov D.M; Minnullin M.M; Farrakhov A.Z. et al. Bioelectrical activity of the gastrointestinal tract in acute intestinal obstruction. // Bulletin of surgery im. I.I. Grekova 2004. Volume 163. No. 1. -p. 25-27.

5. Navruzov S.N; Abdujabbarov S.B; Sapaev D.A. Features of diagnosis and treatment of acute postoperative intestinal obstruction in diseases of the colon. // Medical Journal of Uzbekistan: 2003. №3.-p. 31-33.

6. Navruzov S.N; Mehmonov Sh.R; Dolimov K.S. Extended and combined operations for colonic obstruction. // Bulletin of the Association of Doctors of Uzbekistan: 2003. No. 3. -p. 20-23.

7. Prokhorov G. P; Sidorov A.V; Shaikhelislamova A.O. Double-row precision intestinal suture in conditions of peritonitis and the characteristics of the regeneration of interintestinal anastomoses. // Annals of Surgery 2008. No. 5. -p. 59- 62.

8. Salamov K.N; Zhuchenko A.P; Moskalev A.I. The choice of a method for restoring the natural passage through the colon after Hartman's operation with a "short" rectal stump // Russian Journal of Gastroenterology, Hepatology, Coloproctology. 2001. -№1. p. 56-61.

9. Sysoev S.V. Improved single-row suture in surgery of the small and large intestine (experimental and clinical study) // Abstract of the thesis for the degree of candidate of medical sciences. Ufa: 2009. -pp. 23. 
10. Khodzhimatov G.M; Ten D.O. The results of the formation of the colon anastomosis after resection of the sigmoid colon (experimental study) // Vyatka medical bulletin. 2013. №3. - pp. 17-20.

11. Khodzhimatov G.M; Khamdamov Kh. Kh; Turgunov Sh. Sh; Ten D.O; Akhmedov M.M; Mamadiev A. Experience in the treatment of patients with acute obstruction of the sigmoid colon (experimental clinical study) // Bulletin of emergency medicine. 2013. №4.

- pp. 43-45. 